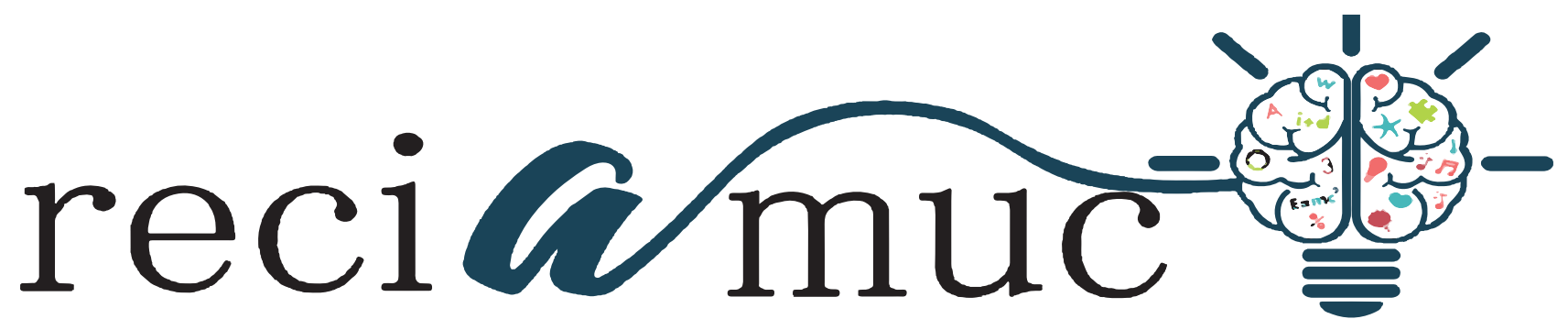

DOI: 10.26820/reciamuc/4.(1).enero.2020.325-332

URL: https://reciamuc.com/index.php/RECIAMUC/article/view/451

EDITORIAL: Saberes del Conocimiento

REVISTA: RECIAMUC

ISSN: 2588-0748

TIPO DE INVESTIGACIÓN: Artículo de Revisión

Códico UNESCO: 3201 Ciencias Clínicas

PAGINAS: 325-332

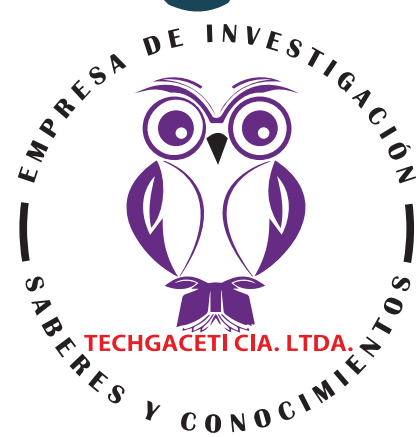

\title{
Cuidados del acceso vascular para hemodiálisis
}

\section{Vascular access care for hemodialysis}

\section{Cuidados de acesso vascular para hemodiálise}

Edison Antonio Rivera Moreira'; Marlon Alberto Franco Fernandez'; Oscar Alexander Enriquez Cali3; Maria Elena Toro Espinoza ${ }^{4}$

RECIBIDO: 18/11/2019 ACEPTADO: 20/12/2019 PUBLICADO: 31/01/2020

1. Médico; Investigador Independiente; Guayaquil, Ecuador; eddie_21_91@hotmail.com; (iD https://orcid. org/0000-0003-0579-7832

2. Médico; Investigador Independiente; Guayaquil, Ecuador; marlon_ff23@hotmail.com; (iD https://orcid.org/00000003-1566-9236

3. Médico; Investigador Independiente; Guayaquil, Ecuador; droscarenriquez@yahoo.com; iD https://orcid. org/0000-0002-9358-2219

4. Médico; Investigador Independiente; Guayaquil, Ecuador; ma.elenatoroe19@gmail.com; (iD https://orcid. org/0000-0003-2474-4621

CORRESPONDENCIA

Edison Antonio Rivera Moreira

maría.velezle@ug.edu.ec

Guayaquil, Ecuador

C C RECIAMUC; Editorial Saberes del Conocimiento, 2020 


\section{RESUMEN}

Una de las bases fundamentales del tratamiento sustitutivo renal en hemodiálisis está constituido por el acceso vascular. Los cuidados que se puedan prestar a los pacientes en las salas de hemodiálisis, así como el autocuidado que el propio paciente o su cuidador puedan tener en su domicilio, son de vital importancia para la eficacia del tratamiento y la mejora de su calidad de vida. Asimismo, se asocia estos cuidados con la mayoría de complicaciones y morbimortalidad de los pacientes. En tal sentido, el objetivo de la presente investigación es plasmar los diferentes cuidados que deben prestarse a los accesos vasculares para hemodiálisis, para lo cual resulta provecho definir los tipos de accesos vasculares y algunas de sus principales complicaciones. El diseño de investigación que se llevó a cabo es de tipo documental o bibliográfico. En los resultados se encontró que los tipos de accesos vasculares para hemodiálisis pueden ser por medio de fístula catéter o injerto. Asimismo, entre las principales complicaciones de los accesos vasculares se encuentran las infecciones, la estenosis y la trombosis. Los cuidados pueden ser prestados tanto por el personal médico como por el propio paciente o cuidador en el hogar y están orientados a mantener vigilada la zona del acceso vascular con la finalidad de detectar y/o evitar algún tipo de complicación. En cualquier caso, éstos cuidados deben estar centrados en la prevención o detección de las complicaciones de los accesos venosos para la hemodiálisis, debiéndose, en primer lugar, llevar una monitorización periódica de estos accesos, así como registrar todas las incidencias presentadas por el acceso y, en último lugar, informar al paciente acerca del cuidado y manejo de su acceso vascular.

Palabras clave: Hemodiálisis, Acceso, Vascular, Cuidados, Catéter.

\section{ABSTRACT}

IOne of the fundamental bases of renal replacement therapy in hemodialysis is constituted by vascular access. The care that can be given to patients in the hemodialysis rooms, as well as the self-care that the patient himself or his caregiver can have at home, are of vital importance for the effectiveness of the treatment and the improvement of their quality of life. Likewise, this care is associated with the majority of complications and morbidity and mortality of patients. In this sense, the objective of the present investigation is to capture the different care that should be given to vascular accesses for hemodialysis, for which it is useful to define the types of vascular accesses and some of their main complications. The research design that was carried out is documentary or bibliographic. In the results it was found that the types of vascular accesses for hemodialysis can be through fistula, catheter or graft. Also, among the main complications of vascular accesses are infections, stenosis and thrombosis. The care can be provided by both the medical staff and the patient or caregiver in the home and are aimed at keeping the area of vascular access monitored in order to detect and / or avoid some kind of complication. In any case, these cares should be focused on the prevention or detection of the complications of venous accesses for hemodialysis, having to, first of all, carry out periodic monitoring of these accesses, as well as recording all the incidents presented by the access and Lastly, inform the patient about the care and management of their vascular access.

Keywords: Hemodialysis, Access, Vascular, Care, Catheter.

\section{RESUMO}

Uma das bases fundamentais da terapia renal substitutiva na hemodiálise é constituída pelo acesso vascular. Os cuidados que podem ser prestados aos pacientes nas salas de hemodiálise, assim como o autocuidado que o próprio paciente ou seu cuidador pode ter em casa, são de vital importância para a efetividade do tratamento e a melhoria de sua qualidade de vida. . Da mesma forma, esse cuidado está associado à maioria das complicações e morbimortalidade dos pacientes. Nesse sentido, o objetivo da presente investigação é captar os diferentes cuidados que devem ser dados aos acessos vasculares para hemodiálise, para os quais é útil definir os tipos de acessos vasculares e algumas de suas principais complicações. O desenho da pesquisa realizado é documental ou bibliográfico. Nos resultados, verificou-se que os tipos de acessos vasculares para hemodiálise podem ser por fístula, cateter ou enxerto. Além disso, entre as principais complicações dos acessos vasculares estão infecções, estenose e trombose. Os cuidados podem ser prestados pela equipe médica e pelo paciente ou cuidador em casa e visam manter a área de acesso vascular monitorada, a fim de detectar e / ou evitar algum tipo de complicação. De qualquer forma, esses cuidados devem ser focados na prevenção ou detecção de complicações dos acessos venosos para hemodiálise, devendo, antes de tudo, realizar um monitoramento periódico desses acessos, além de registrar todos os incidentes apresentados pelo acesso e Por fim, informe o paciente sobre os cuidados e a gestão do seu acesso vascular.

Palavras-chave: Hemodiálise, Acesso Vascular, Cuidados, Cateter. 


\section{Introducción}

El acceso vascular constituye una de las bases del tratamiento sustitutivo renal en hemodiálisis para pacientes con enfermedad crónica renal. Los cuidados que se puedan dispensar a los pacientes en las salas de hemodiálisis, así como el autocuidado que el propio paciente o su cuidador puedan tener en su domicilio, son de vital importancia para la eficacia del tratamiento y la mejora de su calidad de vida. Asimismo, se asocia estos cuidados con la mayoría de complicaciones y morbimortalidad de los pacientes.

"La enfermedad renal crónica afecta a cerca del $10 \%$ de la población mundial. Se puede prevenir, pero no tiene cura, suele ser progresiva, silenciosa y no presentar síntomas hasta etapas avanzadas, cuando las soluciones ya son altamente invasivas y costosas". (Organización Panamericana de la Salud, 2015)

En este orden, Fuentes (2017) señala que, el acceso vascular en el enfermo renal es el sitio anatómico que permite obtener el plasma del torrente circulatorio, y por el que luego es devuelvo una vez este ha pasado por un circuito extracorpóreo de depuración. Específicamente, en hemodiálisis, este tipo conexión se caracteriza por presentar tres características que son: "facilitar el abordaje continuado y seguro al sistema vascular, suministrar flujos suficientes que admitan el suministro de la dosis de diálisis programada y privar complicaciones". (Pág. 29)

En tal sentido, Bodenham (2017) refiere que la asepsia es esencial para todo tipo de accesos vasculares y los cuidados post procedimiento que se deben tener, en razón del acceso directo al torrente sanguíneo que implica. (p. 719)

En líneas generales las medidas o cuidados proporcionados tanto por el personal médico como por el paciente o cuidador, a los diferentes tipos de acceso vascular para la hemodiálisis deben estar dirigidos a reducir el riesgo de complicaciones y a detectarlas de forma temprana.

El objetivo fundamental del presente estudio consiste en plasmar los diferentes cuidados que deben prestarse a los accesos vasculares para hemodiálisis, para lo cual resulta provecho definir los tipos de accesos vasculares y algunas de sus principales complicaciones.

\section{Materiales y Métodos}

La presente investigación se desarrolló mediante una búsqueda de material documental digitalizado, el cual fue recopilado y seleccionado con la finalidad de plasmar el tema de los cuidados del acceso vascular para hemodiálisis, en virtud de lo cual, dicho estudio se clasifica como una investigación de tipo documental o bibliográfica.

Respecto a la metodología aplicada, Ríos, (2017) refiere que la investigación documental "...recurre a la consulta de documentos para obtener sus resultados”. (p. 81).

El material fue localizado a través de algunas bases de datos y páginas web reconocidas científicamente en el área de la salud internacional, entre las que se encuentran: MedlinePlus, PubMed, SciELO, Dialnet, entre otras. Asimismo, se usaron para la búsqueda las siguientes palabras claves o descriptores: "Accesos Vasculares", "Accesos Vasculares para Hemodiálisis", "Hemodiálisis" y "Cuidados del Acceso Vascular para Hemodiálisis". La búsqueda resultó en más de un centenar de miles de registros bibliográficos, filtrados según los criterios de correlación temática, idioma español, actualidad y relevancia.

\section{Resultados}

\section{Tipos de Acceso Vascular}

Existen tres tipos de accesos:

- Fístula arteriovenosa: Una fístula arteriovenosa creada quirúrgicamente es una conexión entre una arteria y una vena, generalmente en el brazo que menos 
usas. Este es el tipo preferido de acceso debido a su eficacia y seguridad. (ver Figura 1)

- Injerto de fístula arteriovenosa: Si tus vasos sanguíneos son demasiado pequeños para una fístula arteriovenosa, el cirujano puede crear una ruta entre una arteria y una vena usando un tubo sintético flexible llamado «injerto».

- Catéter venoso central: Si necesitas hemodiálisis de urgencia, se puede insertar un tubo plástico (catéter) en una vena larga en tu cuello o cerca de la ingle. El catéter es temporal. (Mayo Clinic, 2019)

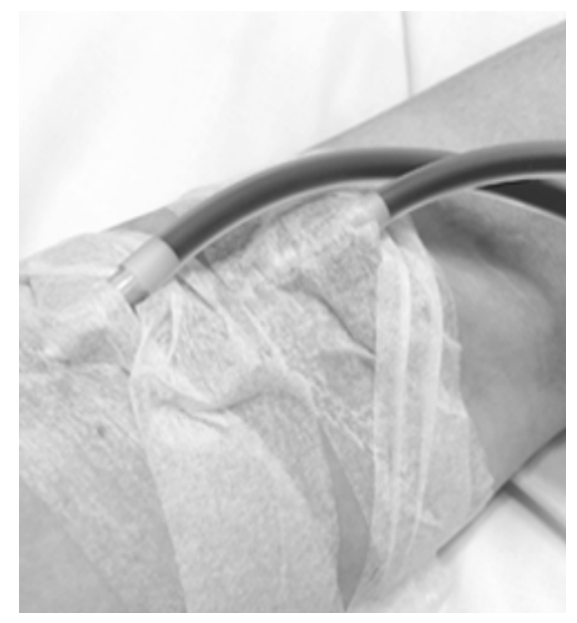

Figura 1. Fístula para Hemodiálisis

Fuente: "Cuidados del acceso vascular para hemodiálisis". Instituto Salvadoreño del Seguro Social. (2017). Recuperado de http://aps.isss.gob.sv/familia/salud\%20al

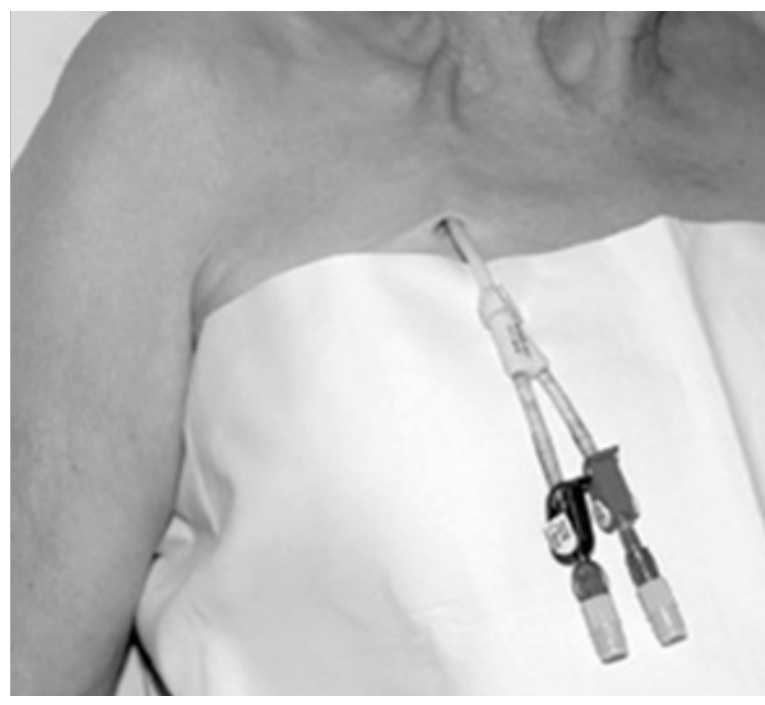

Figura 2. Catéter para Hemodiálisis

Fuente: "Cuidados del acceso vascular para hemodiálisis". Instituto Salvadoreño del Seguro Social. (2017). Recuperado de http://aps.isss.gob.sv/familia/salud\%20al\%20d\%C3\%ADa/Cuidados\%20del\%20acceso\%20vascular\%20para\%20hemodi\%C3\%A1lisis 


\section{Complicaciones del acceso vascular}

Crespo Montero et al. \& Tulleuda et al. citados por Fiterre et al. (2016) exponen consecutivamente que las infecciones son dependientes del tipo de acceso utilizado para la hemodiálisis; así "los pacientes portadores de fístula arteriovenosa (FAV) tienen el menor índice de infección, seguidos del injerto arteriovenoso (AV), luego los catéteres tunelizados y finalmente la mayor incidencia de infección la presentaron los pacientes con catéteres venosos centrales no tunelizados". (p. 344)

La mayoría de las complicaciones de la hemodiálisis vienen dadas por las infecciones que se pueden contraer por medio del acceso vascular. Al respecto, Fiterre et al. (2016) también indican:

Los pacientes tratados con hemodiálisis tienen mayor susceptibilidad a contraer infecciones, favorecidas por la necesidad de un acceso vascular, los intervalos de circulación extracorpórea y las comorbilidades asociadas. Las infecciones, solo precedidas por los eventos cardiovasculares, representan en los pacientes en hemodiálisis la segunda causa de muerte, con una mortalidad atribuible de 14\%, siendo las infecciones de los accesos vasculares la primera causa de bacteriemia. (p. 342)

Cuidado del acceso vascular por parte del personal de enfermería

1. Procedimiento de conexión a hemodiálisis.

- Se procede a informar al paciente sobre la técnica que se va a realizar. Se coloca al paciente en decúbito supino y en ligero Trendelemburg. El personal de salud y el paciente se les colocan mascarillas. Se hace un lavado aséptico de manos, para con posterioridad colocarnos los guantes de un solo uso. Se descubre el catéter de esparadrapos y gasas, y se deposita sobre campo estéril. Se inspecciona la zona de inser- ción del catéter, evaluando si presenta enrojecimiento, exudado, maceración, o por el contrario está limpia. Si se considera necesario, éste es el momento de tomar una muestra para cultivo. Se procede a la cura del punto de inserción con clorhexidina jabonosa, suero fisiológico, clorhexidina y tapamos el punto con apósito trasparente para así poder observar la evolución del orificio.

- Se envuelven las dos luces del catéter en una compresa empapada con el desinfectante apropiado y se deja actuar durante el tiempo necesario. Se desecha los guantes, se procede nuevamente a lavado aséptico de manos, o bien lavado de manos con desinfectante alcohólico y se calzan nuevos guantes. Se prepara otra compresa o gasa empapada en desinfectante para limpiar las luces, procurando que estas queden el menor tiempo posible expuestas al exterior.

- Se retiran los tapones e inmediatamente conectamos una jeringa de $5 \mathrm{cc}$ en cada luz para extraer el sello de heparina que se encuentra en el interior de ambas luces, se aspira unos 5 cc para extraer el posible coagulo que se haya podido formar y los restos de Heparina que queden en la luz del catéter, con ello se comprueba la permeabilidad del mismo.

- Se cambia la jeringa del apartado anterior por una de las de 20 cc con 10 cc de suero fisiológico y se introduce su contenido en las luces del catéter con rapidez. Las conexiones del catéter a las líneas tanto arterial como venosa del circuito de hemodiálisis, se realizan extremando las medidas de asepsia y procurando que la exposición del catéter al exterior sea lo más breve posible. Finalizada la conexión a las líneas, se cubre el catéter y las conexiones con un paño estéril, fijando el sistema para evitar movimientos que se puedan transmitir a la inserción del catéter. 
2. Procedimiento de desconexión de hemodiálisis.

- Se preparan 2 jeringas de 5 cc con suero fisiológico, medicación si está prescrita. Se realizan idénticas maniobras de asepsia y colocación de mascarilla. Se cierra la pinza del catéter, se desconecta la línea arterial y se introducen $5 \mathrm{cc}$ de suero fisiológico, se procede de igual manera con el ramal venoso del catéter una vez finalizado el retorno de la sangre al paciente.

- Se retira la jeringa de $5 c c$ y se introduce la solución deseada para heparinizar.

- Para el sellado, se introduce la cantidad del compuesto según el volumen indicado en el catéter, este paso se realiza siempre cerrando el clamp contra presión. Se retira la jeringa y se tapa con un tapón estéril, maniobra igual para las 2 vías. Se cubre con saco estéril adecuando su posición a la comodidad del paciente y se fija. Se registran las actividades realizadas, así como las recomendaciones que se consideren necesarias. (Hermosín, Pereira, \& Calviño, 2017)

\section{Protocolo de cuidado del catéter venoso central (CVC)}

Garnica et al. (2012) recomiendan en su estudio los siguientes cuidados para los catéteres venosos centrales:

- Debe estar cubierto y seco. Ante alguna incidencia (dolor, calor, rubor...), llamar o acudir a su unidad de diálisis.

- Prevenir infecciones mediante una adecuada higiene, proteger el catéter durante la misma, evitando dirigir el chorro de agua hacia esa zona.

- Evitar objetos cortantes, cadenas gruesas en el cuello, tirantes u objetos que pueda comprimirlo, así como los movimientos bruscos o forzados.

- Si catéter en ingle, no flexionar la pierna excesivamente. (p. 131)

\section{Protocolo de cuidado de la fístula arterio- venosa (FAV)}

Para el cuidado de la fístula arteriovenosa, el paciente debe estar atento y considerar las siguientes recomendaciones:

- Comprobar diariamente el funcionamiento del thrill.

- Si nota disminución o ausencia de latido llame inmediatamente al hospital y que localicen al nefrólogo de guardia.

- Mantener el brazo de la fístula limpio. Lavarlo con agua y jabón y mantener las uñas limpias y cortas.

- Quitarse las tiritas al día siguiente de la diálisis y lavarse el brazo.

- Realizar ejercicios con pelota para favorecer el desarrollo de la misma.

- Piel bien hidratada.

- Si dolor brusco o intenso en la zona de la fístula o sangrado, compresión con los dedos. Si persistiese acudir a su centro de diálisis. (Garnica et al., 2012, p. 131)

\section{Cuidado personal del acceso vascular para hemodiálisis}

El paciente al salir del hospital o clínica puede presentar un poco de enrojecimiento o hinchazón en la zona de acceso sobre todo durante los primeros días. El cuidado básico diario de un acceso vascular para evitar infecciones, coágulos sanguíneos y otros problemas con su acceso vascular, puede incluir:

- Siempre lávese las manos con jabón y agua caliente antes y después de tocar su acceso. Limpie el área alrededor del acceso con jabón antibacterial o alcohol antes de los tratamientos de diálisis.

- Revise el pulso (también llamado frémito) en su acceso todos los días. Su proveedor le mostrará cómo hacerlo.

- Cambie el lugar donde la aguja penetra 
en la fístula o el injerto para cada tratamiento de diálisis.

- NO deje que nadie le tome la presión arterial, le ponga una IV (vía intravenosa) o le saque sangre del brazo donde está el acceso.

- NO deje que nadie le saque sangre de su catéter venoso central con túnel.

- NO duerma sobre el brazo que tiene el acceso.

- NO cargue más de 10 libras (4.5 kilos) con el brazo del acceso.

- NO use reloj, joyas ni ropa ajustada sobre el sitio del acceso.

- Tenga cuidado de no golpear ni cortar su acceso.

- Utilice su acceso solamente para la diálisis. (Enciclopedia Médica ADAM, 2020)

\section{Conclusiones}

Los cuidados del acceso vascular pueden ser brindados tanto por el personal de salud en un centro asistencial como por el propio paciente o algún cuidador en el hogar, y de ellos va a depender el éxito o el fracaso del tratamiento y, por ende, la calidad de vida del paciente.

En el caso de los cuidados prestados por el personal de enfermería, estos son indispensables en la vida de los accesos vasculares de estos pacientes sometidos, son estos profesionales los encargados de explicar a los pacientes el tipo de acceso a practicársele para la hemodiálisis, así como la práctica del mismo y cuidado, dentro del centro de salud.

Por otra parte, los pacientes deben estar bien informados para enfrentar y colaborar con el tratamiento de su enfermedad, de esta forma asumir el papel que le corresponde, ya sea al propio paciente o a un familiar o cuidador, para llevar a cabo los cuidados del acceso vascular que sean pertinentes al caso.

En cualquier caso, éstos cuidados deben estar centrados en la prevención o detección de las complicaciones de los accesos venosos para la hemodiálisis, debiéndose, en primer lugar, llevar una monitorización periódica de estos accesos, así como registrar todas las incidencias presentadas por el acceso y, en último lugar, informar al paciente acerca del cuidado y manejo de su acceso vascular.

\section{Bibliografía}

Bodenham, A. (2017). Acceso Vascular. Revista Médica Clínica Condes, 28(5), 713-726]. Recuperado el 18 de Febrero de 2020, de https://www.elsevier.es/es-revista-revista-medica-clinica-las-condes-202-pdf-S0716864017301141

Enciclopedia Médica ADAM. (04 de Febrero de 2020). MedlinePlus. Recuperado el 08 de Febrero de 2020, de https://medlineplus.gov/spanish/ ency/patientinstructions/000591.htm

Fiterre, I., Suárez, C., Sarduy, R. L., Castillo, B., Gutiérrez, F., Sabournin, N., \& Ivars, E. V. (2016). Factores de riesgo asociados con sepsis del acceso vascular de pacientes en hemodiálisis. Revista Habanera de Ciencias Médicas, 17(2), 335-346. Recuperado el 02 de Febrero de 2020, de http:// scielo.sld.cu/pdf/rhcm/v17n2/rhcm18218.pdf

Fuentes, L. (2017). Cuidados de enfermería en hemodiálisis, tipos de accesos vasculares y calidad de vida. Universidad de Jaén, Enfermería. Jaén: Universidad de Jaén. Recuperado el 01 de 02 de 2020, de http://tauja.ujaen.es/bitstream/10953.1/6200/1/ Fuentes_Gonzlez_Laura_TFG.pdf

Garnica, A., Remón, N., Santesteban, A., Gutiérrez, J. M., Ruiz, L., Zapatería, C., . . González, E. (2012). Protocolo para el cuidado del acceso vascular en pacientes con terapia renal sustitutiva (TRP). Enfermería Nefrología, 15(1), 102-141. Recuperado el 02 de Febrero de 2020, de http://scielo.isciii.es/ pdf/enefro/v15s1/82_poster-hemodialisis23.pdf

Hermosín, A., Pereira, E., \& Calviño, I. (2017). Cuidados de Enfermería en el manejo de catéteres venosos centrales en Hemodiálisis. Revista Médica Electrónica Portales Médicos. Recuperado el 11 de Febrero de 2020, de https://www.revista-portalesmedicos.com/revista-medica/cuidados-enfermeria-cateter-venoso-central-hemodialisis/

Instituto Salvadoreño del Seguro Social - ISSS. (29 de Agosto de 2017). Recuperado el 12 de Febero de

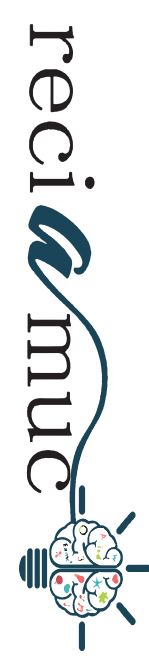


2020, de http://aps.isss.gob.sv/familia/salud\%20 al\%20d\%C3\%ADa/Cuidados\%20del\%20acceso\%20vascular\%20para\%20hemodi\%C3\%A1lisis

Mayo Clinic. (14 de Diciembre de 2019). Mayo Clinic. Recuperado el 30 de Enero de 2020, de https://www.mayoclinic.org/es-es/tests-procedures/ hemodialysis/about/pac-20384824
Ríos, R. (09 de 2017). www.eumed.net. (E. Grupo de investigación (SEJ 309) eumed.net de la Universidad de Málaga, Ed.) Obtenido de www.eumed.net: http://www.eumed.net/libros/libro.php?id=1662

\section{CITAR ESTE ARTICULO:}

Rivera Moreira, E., Franco Fernandez, M., Enriquez Cali, O., \& Toro Espinoza, M. (2020). Cuidados del acceso vascular para hemodiálisis. RECIAMUC, 4(1), 325-332. doi:10.26820/reciamuc/4.(1).enero.2020.325-332

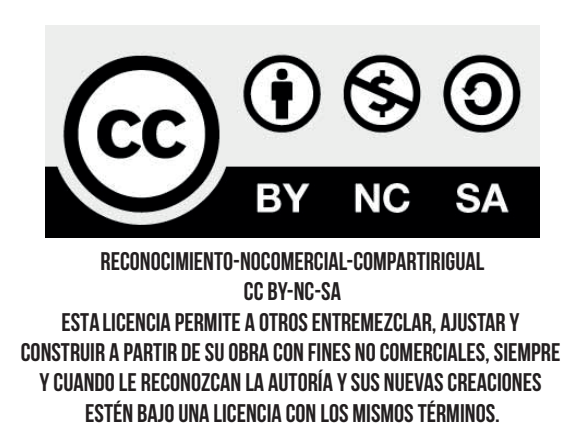

